\title{
A THEORETICAL AND EXPERIMENTAL STUDY OF THE VIBRATION GALVANOMETER.
}

\author{
By Frank Wenner.
}

CONTENTS.

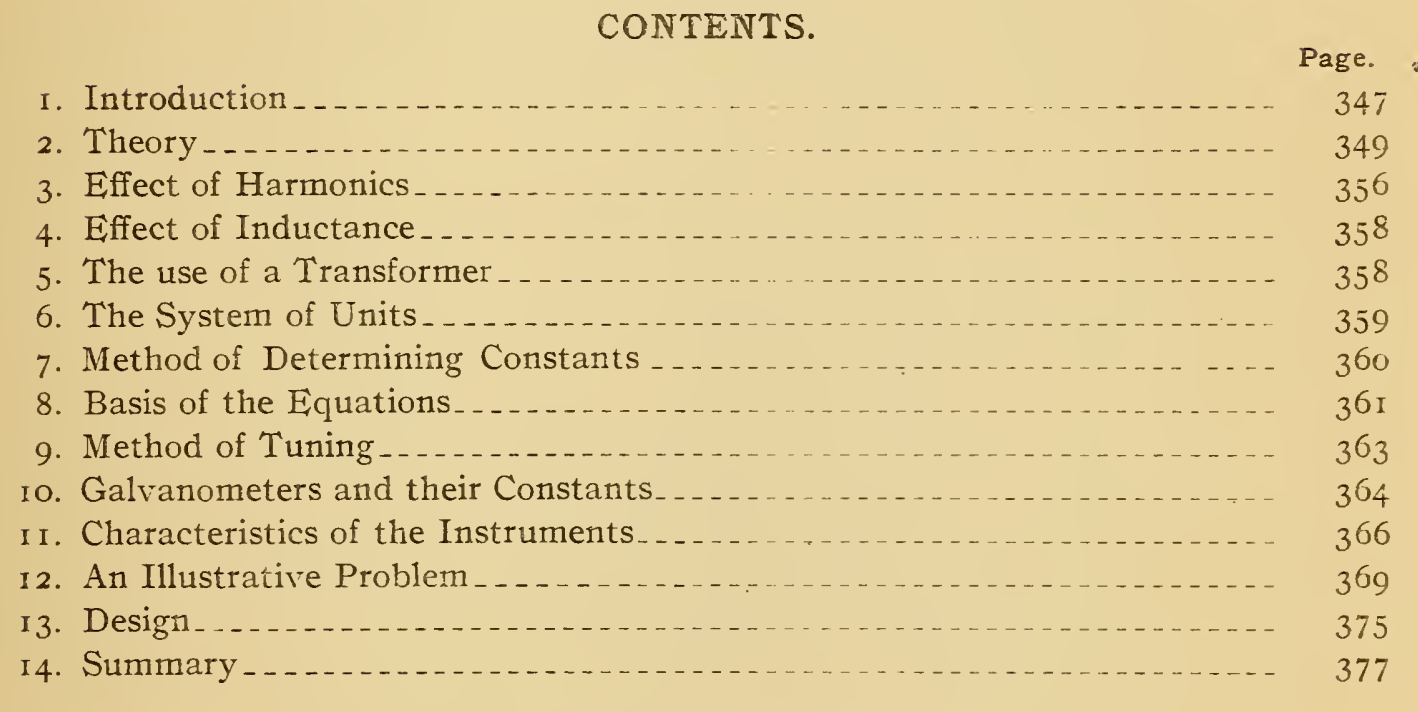

\section{INTRODUCTION.}

The vibration galvanometer is an instrument for the detection of alternating currents or electromotive forces. It differs from other instruments for the same purpose mainly in having the moving system tuned to the frequency of the current or electromotive force to be investigated. In one of the later designs the construction differs from the ordinary D'Arsonval galvanometer only in having a very narrow coil and having bifilar suspensions of adjustable length and tension so as to give a period of from about 50 to about 750 vibrations per second. As the deflection of the instrument changes sign as often as the current changes direction, the image formed by the mirror of a line source of light appears as a band, unless the frequency is very low. The width of the band is a measure of the amplitude of the vibration. 
If the period or frequency of the current is changed while the free period of the instrument is kept constant, it is found that the instrument has a high sensibility at and near the resonating frequency, while at frequencies differing by more than a few per cent from the resonating frequency the sensibility is very small. The instrument, therefore, responds only slightly to the harmonics in the current or electromotive force wave, so that usually no error is introduced when measurements and calculations are made on the assumption of a pure sine wave. This property makes the instrument especially suitable for those measurements in which frequency enters and in which null methods are used.

Max Wien ${ }^{1}$ pointed out some of the advantages of a tuned instrument when he described his optical telephone, a vibration galvanometer in which the essential part of the moving system consists of a piece of soft iron on a very flexible diaphragm. The soft iron armature is actuated by one or two bipolar telephone magnets of the usual construction. The current to be investigated, passing through the coils, causes a relative displacement between the magnet and armature, and this is communicated to a mirror in such a way as to give an angular displacement to the latter. The displacement is read by the broadening of a line image. The mirror is on a spring, and for a maximum sensibility the period of this system, as well as the period of the current to be investigated, is adjusted to the period of the diaphragm. Wien showed experimentally that the deflection is proportional to the current.

Rubens ${ }^{2}$ next brought out an instrument in which the mirror and soft iron piece are mounted together on a metal suspension instead of a diaphragm. The bipolar telephone magnets are so placed and their coils are so connected that the current causes an angular displacement of the soft iron armature and mirror. The frequency can be adjusted by either a change in length or tension of the suspensions. This instrument is more flexible than the optical telephone and a somewhat higher sensibility is claimed for it.

Max Wien ${ }^{3}$ later developed an instrument having a very small permanent magnet mounted on a brass wire suspension, the

\footnotetext{
${ }^{1}$ Wied. Ann., 42, p. 593, and 44, p. 68I; 1891.

${ }^{2}$ Wied. Ann., 56, p. 27 ; 1895 .

${ }^{3}$ Ann. der Physik., 309, p. 425; 1901 .
} 
lengths and tension of which may be changed so as to give a considerable range in the number of vibrations per second. The coils are wound on a core of soft iron wire, and the pole faces of this core are very close and parallel to the small magnet. The sensibility claimed for this instrument is 16 times that of the optical telephone.

Rosa and Grover ${ }^{4}$ in their work on the measurement of inductance pointed out certain advantages in the use of a vibration galvanometer and give a curve showing the change in sensibility with a change in tuning for an instrument which apparently had two free periods.

Wells ${ }^{5}$ showed experimentally that the deflection is proportional to the electromotive force. He also plotted a curve showing the change in the observed deflection as the free length of the suspension was varied.

In 1907 Campbell ${ }^{6}$ described a very convenient instrument of the D'Arsonval type. This instrument is suitable for frequencies of from about 50 to about 750 vibrations per second and is easily adjustable over this range. In sensibility this instrument compares favorably with Wien's when both are adjusted to the same frequency. Concerning the theory of the instrument, Campbell points out that the sensibility is independent of the moment of inertia and inversely proportional to the frequency. He also states that the damping is "both mechanical and electrical" and should be kept small.

\section{THEORY.}

In any galvanometer, a current through the winding produces a force tending to cause a relative displacement between the fixed and moving system. The resulting relative displacement causes a part of the flux from the magnet to cut through the winding, and thus develops a counter electromotive force. The energy available for producing a deflection is the time integral of the product of the current and the counter electromotive force. As galvanometers are ordinarily used, this energy is small, due to the

${ }^{4}$ This Bulletin, 1, p. 291; 1905:

${ }^{5}$ Phys. Rev., 23, p. 504; 1906.

${ }^{6}$ Phil. Mag., 14, p. 494; 1907.

Electrician, 60, p. 60; 1907. 
fact that after the moving system has reached a displacement equal to the final deflection the time integral of the product of the counter electromotive force and current is either equal to or less than zero.

If the motion is only slightly damped, a reversal of the current at the end of the first deflection will cause a second deflection in the opposite direction larger than the first. This is due to the fact that the motion of the system again causes a counter electromotive force, and thus more electrical energy is converted into mechanical energy. However, not all this energy is added to the. system, since some is lost by air friction, induced currents, etc. By reversing the current at the end of each deflection the successive deflections can be increased until the average rate at which energy is lost by damping becomes equal to the average rate at which electrical energy is converted.

In the work that follows we shall consider first the case where the impressed electromotive force and current waves are both of a sine form and later the effect of harmonics. With a sine wave current and electromotive force we may assume a simple harmonic vibration, and a sine wave back electromotive force.

In use the free period is adjusted to or near that of the current to be investigated. If this adjustment is good the displacement of the moving system leads the driving current by $90^{\circ}$, and since the back electromotive force depends upon the rate of change of this displacement it is in phase with the current. If, however, the tuning is not good the moving system leads the current by an angle greater or less than $90^{\circ}$, and consequently the phase angle between the current and the back electromotive force is not zero. As the power converted depends upon the cosine of this phase angle, the tuning may have a marked effect upon the deflection.

As the amplitude of the vibration increases the back electromotive force increases and unless the resistance is very high it has a marked effect on the value of the current. If the three vectorsthe current, impressed electromotive force, and back electromotive force-are in the same line, the power converted and consequently the deflection is a maximum, if after a steady condition is reached the back electromotive force is equal to one-half the impressed electromotive force. Since the back electromotive force is pro- 
portional to the field strength it may happen that an increase in the field strength results in a decrease in the amplitude, just as in a direct-current motor an increase of the field strength may result in a decrease in the speed. For a given set of conditions the deflection of the vibration galvanometer is a maximum for a particular field strength, just as for a given set of conditions the speed of a direct-current motor is a maximum for a particular field strength.

Since when a steady condition is reached the power lost by damping is equal to the electrical power converted, a decrease in the damping must, if the other quantities are kept fixed, result in an increase in the amplitude of the vibration. This increase may or may not be large, just as in the direct-current motor a decrease in the load may or may not result in a material increase in the speed.

The relations between the amplitude of the vibration, current, electromotive force, frequency, etc., may be expressed in terms of the working constants, the more important of which are: $(a)$ The current sensibility, (b) the electromotive force sensibility, $(c)$ the power sensibility, $(d)$ the ratio of the back to the impressed electromotive force, $(e)$ the time constant for current indication, $(f)$ the time constant for electromotive force indication, $(g)$ the resonance range for current indication, and $(h)$ the resonance range for electromotive force indication. By resonance range is meant the fractional part by which the period of the current or the impressed electromotive force is changed to reduce the deflection by half.

These working constants depend upon the intrinsic constants of the instrument which are: (a) The moment of inertia, (b) the moment of damping (by which is meant the ratio of the retarding torque to the angular speed when the coil is on open circuit), (c) the moment of restoration (the ratio of the torque, tending to bring the moving system to the null position, to the angular displacement), $(d)$ the moment of displacement (the ratio of the torque produced by the current to the current), and (e) the resistance.

The intrinsic constants as here given and defined refer only to an instrument in which the displacement is angular. The following equations, however, are not limited to this kind of a displacement but are equally applicable (except for the constant where an 
arbitrary unit of displacement is chosen) to instruments of the Einthoven or string type.

The equations giving the relation between the various intrinsic constants, the deflection, time, and current or electromotive force will be stated and solved under certain limiting conditions. The symbols used are as follows:

$\theta=$ angular displacement.

$t=$ time.

$\alpha=$ moment of inertia.

$\beta=$ moment of damping.

$\gamma=$ moment of restoration.

$\psi=$ moment of displacement.

$\rho=$ resistance of galvanometer.

$r=$ resistance of complete galvanometer circuit.

$i=$ instantaneous value of current.

$I^{\prime}=$ maximum value of current.

$I=$ effective value of current.

$w=$ power.

$f=$ frequency of current or impressed electromotive force.

$f_{o}=$ natural free frequency of moving system.

$p=2 \pi f$.

$p_{0}=2 \pi f_{0}$.

$\phi=$ amplitude after a steady condition is reached.

$e=$ instantaneous value of the back electromotive force.

$E=$ effective value of impressed electromotive force.

$E_{b}=$ effective value of back electromotive force.

$E_{o}=$ effective value of impedance electromotive force.

$\omega=$ the angle by which the impressed electromotive force leads the current.

$\eta=$ the angle by which the impedance electromotive force leads the current.

$\sigma=$ the angle by which the back electromotive force leads the current. ${ }^{7}$

$A=$ current sensibility.

$V=$ electromotive force sensibility.

$A^{\prime}=$ direct current sensibility.

${ }^{7}$ When the electromotive force developed in the winding of the instrument is in exact opposition to the current $\sigma$ is taken as zero as shown in Fig. I. 
$W=$ power sensibility.

$D=$ ratio of back to impressed electromotive force.

$T_{i}=$ time constant for current indications.

$T_{e}=$ time constant for electromotive force indications.

$R_{e}=$ resonance range for electromotive force indications.

$R_{i}=$ resonance range for current indications.

$X=$ the step-up ratio of the transformer.

$r_{1}=$ resistance of primary circuit.

$V_{x}=$ electromotive force sensibility with transformer.

- signifies that the quantity in question is referred to the set of conditions given on page 359 .

The motion of the moving system of a galvanometer is given ${ }^{8}$ by the equation

The free period

$$
\alpha \frac{d^{2} \theta}{d t^{2}}+\beta \frac{d \theta}{d t}+\gamma \theta=\psi i
$$

or

$$
\mathrm{I} / f_{o}=2 \pi \sqrt{\alpha / \gamma}
$$

and if

$$
\begin{gathered}
\gamma / \alpha=p_{o}{ }^{2} \\
i=I^{\prime} \cos p t \\
\frac{d^{2} \theta}{d t^{2}}+\frac{\beta d \theta}{\alpha d t}+p_{o}^{2} \theta=\frac{\psi}{\alpha} I^{\prime} \cos p t
\end{gathered}
$$

Integrating (2) gives

$$
\theta=\frac{\psi I^{\prime} \sin (p t+\sigma)}{\sqrt{p^{2} \beta^{2}+\alpha^{2}\left(p_{o}^{2}-p^{2}\right)^{2}}}-C \epsilon^{-\frac{\beta t}{2 a}} \sin \left[\sqrt{p_{o}^{2}-\beta^{2} / 4 \alpha^{2}} t+C^{\prime}\right]
$$

where $C$ and $C^{\prime}$ are constants of integration and

$$
\sigma=\tan ^{-1} \frac{\alpha\left(p_{o}^{2}-p^{2}\right)}{\beta p}
$$

A short time after starting the current the second member of equation (3) becomes zero, hence

$$
\phi=\frac{\sqrt{2} \psi I}{\sqrt{p^{2} \beta^{2}+\alpha^{2}\left(p_{0}^{2}-p^{2}\right)^{2}}}
$$

where $\phi$ is the maximum value of $\theta$ or the amplitude of the vibration.

${ }^{8}$ Gray, Abs. Meas. in Elec. and Mag., Vol. II, Part II, pp. 392-3. 
The current is determined by the components of the electromotive forces in phase with it and the resistance or

$$
I=\frac{E \cos \omega-E_{b} \cos \sigma}{r} .
$$

The rate at which mechanical energy is supplied to the moving system is equal to the rate at which electrical energy is converted.

ig. 1.-Vector Diagram showing the Various Phase Angles and Relations between the Electromotive Forces.

The former is the torque [due to the current and equal to $\psi$ i] times the angular speed $[\phi p \cos (p t+\sigma)]$ and the latter is the current times the back electromotive force. Therefore

$$
i \psi \phi p \cos (p t+\sigma)=i e
$$

or

$$
E_{b}=\psi \phi p / \sqrt{2}
$$

The substitution of the values of $I$ from (5) and $E_{b}$ from (6) in (4) gives

$$
\phi=\frac{\sqrt{2} \psi E \cos \omega}{r \sqrt{p^{2} \beta^{2}+\alpha^{2}\left(p_{0}^{2}-p^{2}\right)^{2}+p \psi^{2} \cos \sigma}}
$$

and the multiplication of (4) by (7) gives

$$
\phi^{2}=\frac{2 \psi^{2} I E \cos \omega}{r\left[p^{2} \beta^{2}+\alpha^{2}\left(p_{0}^{2}-p^{2}\right)^{2}\right]+\sqrt{p^{2} \beta^{2}+\alpha^{2}\left(p_{o}^{2}-p^{2}\right)^{2}} p \psi^{2} \cos \sigma} .
$$

Or if $p=p_{o},(4),(7)$, and (8) become, since $\sigma$ is then zero,

$$
\phi=\frac{\sqrt{2} \psi I}{p \beta}, \quad \phi=\frac{\sqrt{2} \psi E \cos \omega}{p\left(r \beta+\psi^{2}\right)}, \quad \text { and } \phi^{2}=\frac{2 \psi^{2} I E \cos \omega}{p^{2} \beta\left(r \beta+\psi^{2}\right)}
$$


Defining the current sensibility as the ratio of the amplitude of the vibration to the effective value of the current, the electromotive force sensibility as the ratio of the amplitude to the effective value of the electromotive force at the terminals of the instrument, and the power sensibility as the ratio of the square of the amplitude to the power supplied gives

$$
A=\frac{\sqrt{2} \psi}{p \beta}, \quad V=\frac{\sqrt{2} \psi}{p\left(\rho \beta+\psi^{2}\right)}, \quad \text { and } W=\frac{2 \psi^{2}}{p^{2} \beta\left(\rho \beta+\psi^{2}\right)} .
$$

In the second part of (Iо) $\cos \omega$ is taken as unity. This may generally be done without introducing an appreciable error, since in most instruments the impedance electromotive force, between terminals, is small in comparison with and very nearly in phase with the impressed electromotive force.

The time constant is given for current indications by equation (3) or

$$
T_{i}=\frac{2 a}{\beta} .
$$

For electromotive force indications the back electromotive force is zero when $t$ is zero and increases directly with the amplitude of the vibration. The current therefore starts with a certain value and decreases as the amplitude increases, which causes the amplitude to reach a certain fraction of its final value in less time than with a constant current. If the instrument is tuned so that the back electromotive force is in phase with the current it may be shown that the retarding torque is $\frac{r \beta+\psi^{2}}{r} \frac{d \theta}{d t}$ instead of $\beta \frac{d \theta}{d t}$ as given by equation (I) for current indications. It therefore follows that

$$
T_{e}=\frac{2 \kappa r}{r \beta+\psi^{2}}
$$

Since the resonance range is the change, in proportional parts, in the impressed frequency from resonance, necessary to reduce 
the deflection by half, it may be derived from equations (7) and (9) by equating the second member of the first to half the second member of the latter, or

$$
\frac{\sqrt{2} E \psi \cos \omega}{\sqrt{\beta^{2} p^{2}+\alpha^{2}\left(p_{o}^{2}-p^{2}\right)^{2}}+p \psi^{2} \cos \sigma}=\frac{\sqrt{2} E \psi}{2 p_{o}\left(r \beta+\psi^{2}\right)} .
$$

Then if we write $p_{o}(\mathrm{I}+R)$ for $p$, we have, with a close approximation, $4 p_{0}{ }^{4} R^{2}=\left(p_{0}{ }^{2}-p^{2}\right)^{2}$, which when substituted in (I 3 ), and writing $p_{o}$ for $p$, where it makes practically no difference, gives

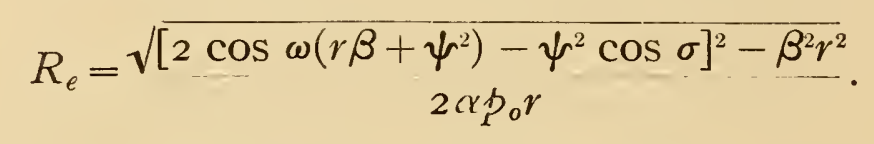

Here, since there is a decided lack of tuning, $\cos \sigma$ is small and in comparison with 2 may be neglected while $\cos \omega$ has a value between .8 and $\mathrm{I}$. Taking the latter gives

$$
R_{e}=\frac{\sqrt{3 \beta^{2} \gamma^{2}+8 \beta r \psi^{2}+4 \psi^{4}}}{2 \alpha r p_{o}}
$$

a value about 20 per cent too large if the resistance is small. If the resistance is very large, the back electromotive force has no effect on the value of the current, or only those terms of (I5) involving the resistance are of importance, so that

$$
R_{i}=\frac{\sqrt{3} \beta}{2 \alpha p_{0}} .
$$

\section{EFFECT OF HARMONICS.}

In those measurements in which the frequency enters and null methods are used an exact balance can not be obtained except when the electromotive force and current waves are of a pure sine form. In practice this condition is seldom if ever fulfilled, so the vibrations caused by the harmonics may limit the precision attainable. One of the chief characteristics of the vibration galvanometer is that the amplitude of the various harmonic vibrations is small. This is because there is no resonance, and the turning moment changes direction at intervals short in comparison with the half free period of the moving system. The amplitude of the vibration produced by any particular harmonic in the electromo- 
tive force wave is given by equation (7) when the corresponding value of $p$ is substituted.

The substitution of $3 p_{0}$ for $p$ gives

$$
\phi_{3}=\frac{\sqrt{2} \psi E_{3} \cos \omega_{3}}{r \sqrt{9 \beta^{2} p_{o}^{2}+64 \alpha^{2} p_{o}^{4}}+3 p_{o} \psi^{2} \cos \sigma_{3}} .
$$

Here, unless the reactance is large, $\left(\cos \omega_{3}\right)$ may be taken as unity, while the terms in the denominator are all small in comparsion with $8 \mathrm{r} \alpha \mathrm{po}_{0}^{2}$, so we may write

$$
\phi_{3}=\frac{\sqrt{2} \psi E_{3}}{8 r \alpha p_{o}^{2}} .
$$

Defining the sensibility in the usual way

$$
V_{3}=\frac{\sqrt{2} \psi}{8 r \alpha p_{0}^{2}} .
$$

Likewise

$$
\begin{aligned}
& V_{5}=\frac{\sqrt{2 \psi}}{24 r \alpha p_{0}^{2}}, \\
& V_{7}=\frac{\sqrt{2} \psi}{48 r \alpha p_{0}^{2}}, \\
& V_{9}=\frac{\sqrt{2} \psi}{80 r \alpha p_{0}^{2}} .
\end{aligned}
$$

Corresponding substitutions in (4) give

$$
\begin{aligned}
& A_{3}=\frac{\sqrt{2} \psi}{8 \alpha p_{0}^{2}}, \\
& A_{5}=\frac{\sqrt{2} \psi}{24 \alpha p_{0}^{2}}, \\
& A_{7}=\frac{\sqrt{2} \psi}{48 \alpha p_{0}^{2}}, \\
& A_{9}=\frac{\sqrt{2} \psi}{80 \alpha p_{0}^{2}} .
\end{aligned}
$$




\section{EFFECT OF INDUCTANCE.}

By reference to equation (7) it will be seen that the denominator can be decreased (if $\psi$ is large in comparison with $r \beta$ ) by a decrease in $\cos \sigma$, even though in so doing the first term is increased, as it necessarily is when the instrument is thrown slightly out of resonance. If at the same time $\cos \omega$ can be kept near unity, the deflection will be increased. The analytical expression for $\cos \omega$ is complicated, since it involves the back electromotive force and consequently the amplitude of the vibration. However, referring to the vector diagram, Fig. I, it will be seen that if a large inductance is placed in the galvanometer circuit, so as to make the angle $\eta$ nearly $90^{\circ}$, and the frequency of the impressed electromotive force is increased slightly, so that the angle $\sigma$ assumes a negative value somewhat less than $90^{\circ}$, it is possible for the back electromotive force to be much larger than the impressed electromotive force and the latter still have the larger component in phase with the current. The combination of the inductance and the slightly high frequency of the impressed electromotive force result in bringing the back and impressed electromotive force somewhere near quadrature and the latter somewhere near the phase of the current. If the frequency of the impressed electromotive force is not too much above the resonating frequency, the result may be an increase of the component of the current in phase with the impressed electromotive force. This means an increase in the power supplied to and consequently an increase in the amplitude of the vibration of the moving system. A condenser in series with the galvanometer and the lack of tuning in the opposite sense should give corresponding results.

\section{THE USE OF A TRANSFORMER.}

If the terminals of the galvanometer are connected to one of the windings of a transformer and the other winding is connected to the source of electromotive force, the equation for the current in the galvanometer is

$$
I_{2}=\frac{X E-I_{1} X r_{1}-E_{b}}{\rho}=\frac{X E-E_{b}}{\rho+X^{2} r_{1}} .
$$


Here it is assumed that the currents in the transformer windings are in opposition and have the inverse ratio of the number of turns in the coils. The requirements of a transformer to meet these conditions will be considered later. A comparison of equation (20) with equations (5) and (7) gives

$$
\phi=\frac{\sqrt{2} X \psi E \cos \omega}{\left(\rho+X^{2} r_{1}\right) \sqrt{\beta^{2} p^{2}+\alpha^{2}\left(p_{o}^{2}-p^{2}\right)^{2}}+p \psi^{2} \cos \sigma}
$$

or if $p=p_{o}$ and taking $\cos \omega=\mathrm{I}$

and

$$
\phi=\frac{\sqrt{2} X \psi E}{p_{o}\left[\left(\rho+X^{2} r_{1}\right) \beta+\psi^{2}\right]}
$$

$$
\begin{gathered}
V_{x}=\frac{\sqrt{2} X \psi}{p_{0}\left[\left(\rho+X^{2} r_{1}\right) \beta+\psi^{2}\right]} . \\
V_{x} \text { is a maximum when } X^{2}=\frac{\rho \beta+\psi^{2}}{r_{1} \beta} .
\end{gathered}
$$

\section{THE SYSTEM OF UNITS.}

In expressing the sensibility or deflection it will be convenient to use as the unit of current the microampere, of electromotive force the microvolt, of power the micro-microwatt, of deflection a millimeter broadening of a line image one meter in front of a plane mirror ( $=25 \times 10^{-5}$ radians), and cgs units for the other quantities.

The resistance must then be expressed either in ohms or electromagnetic units to correspond with the quantities with which it is associated.

In the following work when the sensibility is expressed in the arbitrary units or the resistance in ohms, it is indicated by a line over the symbol representing the quantity.

Referred to these conditions and writing $2 \pi f_{0}$ for $p_{o}$, we have from equations (IO), (I I), (I 2), and (22), respectively,

$$
\begin{aligned}
& \bar{A}=\frac{A \times \mathrm{IO}^{-7}}{25 \times \mathrm{IO}^{-5}}=A \times 4 \times \mathrm{IO}^{-4}=9.0 \times \mathrm{IO}^{-5} \frac{\psi}{\hat{f}_{0} \beta} \\
& V=V \times 4 \times 1 \mathrm{IO}^{5}=9.0 \times \mathrm{IO}^{4} \frac{\psi}{\hat{f}_{0}\left(\rho \beta+\psi^{2}\right)}
\end{aligned}
$$




$$
\begin{aligned}
& \overline{A^{\prime}}=A^{\prime} 4 \times \mathrm{IO}^{-4}=4 \times \mathrm{IO}^{-4} \frac{\psi}{\gamma} \\
& \bar{W}=W \times 160=\frac{8 . \mathrm{I} \psi^{2}}{f_{o}{ }^{2} \beta\left(\rho \beta+\psi^{2}\right)} \\
& \bar{V}_{x}=\frac{9.0 \times 10^{4} X \psi}{f_{o}\left[\left(\rho+X^{2} r_{1}\right) \beta+\psi^{2}\right]} .
\end{aligned}
$$

\section{METHOD OF DETERMINING CONSTANTS.}

If it is desired to make a change in one or more of the working constants, it is well to determine the intrinsic constants first; then a comparison with the above equations will suggest the changes to make and give the effect of any particular change. The intrinsic constants can all be determined from five independent measurements. Measurements which are easily made and from which the constants can readily be calculated are: $(a)$ the resistance, $(b)$ the direct-current sensibility, $(c)$ the current sensibility, $(d)$ the electromotive force sensibility, and $(e)$ the resonating frequency.

(a) The resistance can be compared directly with a standard by means of a Wheatstone bridge or by other convenient methods.

(b) The direct-current sensibility can be obtained by passing a small known direct current through the coil and observing the change in deflection when the current is reversed.

(c) The galvanometer is then connected through a high resistance to the terminals of a suitable low resistance forming a part of the external circuit of an alternating-current generator. Means should be provided for regulating the speed of the generator. The frequency of the generator or the free period of the instrument is then adjusted so the tuning is good. The resistance shunted, the current through the shunt, and the resistance in series with the galvanometer gives the current, which, with the observed deflection, gives the current sensibility.

(d) The electromotive force sensibility is obtained by shunting a smaller resistance, and the removal of the resistance in series with the galvanometer. The electromotive force is then known

\footnotetext{
${ }^{9}$ Defining the direct-current sensibility in the usual way, the relation follows from equation (I), since when the system has come to rest $\theta \gamma$ or $\phi \gamma=\psi i$. The deflection used is that produced on reversing the current or the double deflection to correspond to the broadening of the image produced by an alternating current
} 
in terms of the shunt and the current through it. This, with the observed deflection, gives the electromotive force sensibility.

(e) The resonating frequency can be obtained from the speed of the generator, considering, of course, the number of pairs of poles.

Reference to equations (23), (23a), and (24) and remembering that $p_{o}{ }^{2}=\gamma / \alpha$, then gives, in terms of the five quantities given above, the intrinsic constants, as follows:

$$
\begin{aligned}
& \alpha=\frac{.9 \mathrm{I}(A-\overline{V \rho})}{f_{0}^{3} \overline{A A^{\prime} V}}, \quad \beta=\frac{8 . \mathrm{I}(\bar{A}-\overline{V \rho})}{f_{0}^{2} \bar{A}^{2} \bar{V}}, \\
& \gamma=\frac{36(\bar{A}-\overline{V \rho})}{f_{0} \overline{A A^{\prime} V}}, \quad \psi=\frac{90000(\bar{A}-\overline{V \rho})}{f_{0} \overline{A V}} .
\end{aligned}
$$

\section{BASIS OF THE EQUATIONS.}

In the derivation of the equations, it has been assumed that the moving system experiences an angular acceleration proportional to the resultant torque, a retarding torque proportional to the angular speed, a restoring torque proportional to the angular displacement, and a displacing torque proportional to the current and independent of the displacement and the rate of change of the current. That is, (a) the moment of inertia, $(b)$ the moment of damping, $(c)$ the moment of restoration, and $(d)$ the moment of displacement are all constant.

It is further assumed that harmonics in the electromotive force or current wave produce no effect on the behavior of the instrument toward the fundamental, but cause an additional vibration depending upon their magnitude and frequency. It should be mentioned that the effect of the time constant of the circuit, which is generally small in comparison with the time constant of the instrument, has been neglected. If the time constant of the circuit is large it may materially effect the rate of change of the amplitude of the vibration.

The moment of inertia and the moment of restoration can better be considered together. If the vibration takes place about the center of the mass the moment of inertia is constant, and for small displacements the restoring moment is at least very nearly proportional to the displacement. When, however, the system is unsymmetrical the vibration, because of an unbalanced lateral 
force due to the tension of the suspensions, takes place about some point or line which does not coincide with or pass through the center of mass and whose position is a function of the frequency of the driving force. Under these conditions the moment of inertia and the moment of restoration are both functions of the frequency of the driving force or current and the system has two free periods. In the article ${ }^{10}$ referred to above, Rosa and Grover found that as the frequency of the current was changed, one of the galvanometers used by them gave maximum deflections at about I I0.5 and I 20 cycles per second, and a very small deflection at I 5 cycles. This shows that the instrument had two free periods differing from each other by about io per cent. This large difference in the periods was caused by a lack of symmetry introduced on the repair of a broken suspension.

If care has been taken to keep the system symmetrical, or if it is carefully balanced, the two free periods may be very nearly the same, but when a few hundredths of a per cent change in the frequency produces a material effect on the deflection, it is possible that the difference in the periods may still be sufficient to produce an appreciable reduction in the sensibility and flattening of the curve, showing the relation between the deflection and the frequency. A little experience enables us to tell, in most cases, from the vibrations produced on tapping the instrument, if the two periods are nearly the same, and, if not, what changes should be made. If, then, the suspensions are bifilar and provided with a means of independent fine adjustment, the two periods can usually be adjusted in a few minutes to very nearly the same value.

The moment of damping is made up of a number of different factors, among which the air pressure and friction, eddy currents and hysteresis are probably the more important. The retarding torque is therefore only approximately proportional to the angular speed, so the moment of damping may depart appreciably from a constant.

The displacing torque is, to some extent, a function of the displacement and is effected by hysteresis and eddy currents. The latter makes the value smaller for alternating than for direct currents, so the moment of displacement is not quite a constant. 
The equations given above, derived on the supposition of these quantities being constant, therefore should not be expected to give exact relations. They should, however, indicate fairly well how an instrument of good design and careful construction will behave under any ordinary set of conditions. A comparison of observed and calculated values in the following work gives in many cases differences no greater than the experimental errors.

\section{METHOD OF TUNING.}

The metnod of tuning first used consists of applying to the terminals of the galvanometer a small alternating electromotive force. and adjusting its frequency or the free period of the moving system until a maximum deflection is obtained. This is the method usually used and for some purposes it is very satisfactory. In general, however, the change in amplitude of the vibration with a change in tuning is small, especially near resonance. If the tuning is made in this way, and observations taken for the electromotive force and current sensibility, a correct value is obtained for the former, but there may be an error of 50 per cent or more in the latter.

If, however, a high resistance (of the order of $100,000 \mathrm{ohms}$ ) is placed in series with the galvanometer, an electromotive force of suitable value used, and the adjustment made for a maximum deflection, the tuning is very much better. This gives a sufficient accuracy in the tuning for most purposes, including the determination of the constants.

However, even with the high resistance a sufficient precision in the tuning could not be obtained for some of the work. It was therefore necessary to use some other method, and the one chosen makes use of the phase angle between the displacement of the moving system and the driving force. On the shaft of the generator is mounted a large disk back of and slightly above which is placed the line source of light. The disk cuts off about half of that portion of the line which it is desired to use except when exposed by notches, one for each pair of poles, cut in the edge. These notches are so placed with respect to the windings that they expose the lower half of the line only at the instant when the electromotive force is a maximum. Relatively large resist- 
ances are used, especially in the galvanometer circuit, so that the resonance curve is sharp and the current may be assumed to be in phase with the electromotive force. One observes then a band of light above and a line of light below the edge of the disk. When the tuning is good, the line appears below the center of the band. When it is disturbed, the line moves toward one side or the other, depending upon the sign of the disturbance, and the displacement is considerable before the shortening of the band is appreciable. A glance at the image tells at once the sign and gives an indication of the magnitude of the lack of tuning so that the adjustment can easily and quickly be made. By using a large resistance in series with a galvanometer having a small moment of damping, this method indicates a lack of resonance when the difference in the frequencies is of the order of o.or per cent of the resonating frequency.

\section{GALVANOMETERS USED AND THEIR CONSTANTS.}

The galvanometers used were one of the Rubens type made by W. Oemke, one of the Campbell type made by Robert W. Paul, and one built in the Bureau of Standards' instrument shop. In what follows the galvanometers are referred to as Nos. I, 2, and 3 in the order here given. The latter was designed with the idea of getting an accurately balanced system of known moment of inertia and of investigating the effect of air pressure. The general construction is somewhat like the Rubens type, except that a single magnet is used. The pole pieces are of transformer iron, and the winding consists of four coils. The moving system consists of a disk of transformer iron $10.5 \mathrm{~mm}$ in diameter and $0.36 \mathrm{~mm}$ in thickness, a circular mirror $10 \mathrm{~mm}$ in diameter and $0.58 \mathrm{~mm}$ in thịckness, and bifilar suspensions of fine steel wire, the latter provided with a means for the independent adjustment of their tension so that any lack of symmetry may be compensated. As arranged, the suspensions form a coil of a single turn which, vibrating in a strong magnetic field, causes a considerable damping. This, however, is of little consequence, since for the present purpose we are not concerned particularly with the sensibility. The case is arranged so that it may be closed tightly and the air exhausted. The fine adjustment of the tuning is made by a soft iron shunt to the magnet. This shunt is on the outside of the case, so that a considerable 
change in the period can easily be made even while the moving system is in a vacuum. Unless otherwise stated it will be understood that the case is open.

The constants of these galvanometers are tabulated in Table I.

\section{TABLE I.}

Constants of the Galvanometers. ${ }^{11}$

\begin{tabular}{|c|c|c|c|c|c|c|}
\hline Galvanometer No. & & I & & 2 & 3 & \\
\hline Alternating current sensibility. . & 1.5 & & 7.3 & & 4.2 & \\
\hline Direct current sensibility .. & .018 & & .010 & & $5.5 \times 10^{-4}$ & \\
\hline EMF sensibility..... & .0014 & & .0061 & & .0075 & \\
\hline Resonating frequency. . & 100 & & 100 & & 100 & \\
\hline Resistance.. & 234 & & 30 & & 74 & \\
\hline $3 d$ harmonic current sensibility. & & .0030 & & .0017 & $9.2 \times 10^{-4}$ & $6.7 \times 10^{-1}$ \\
\hline 3d harmonic EMF sensibility.. & & $13 \times 10^{-6}$ & & $5.6 \times 10^{-6}$ & $9.6 \times 10^{-6}$ & $8.5 \times 10^{-6}$ \\
\hline 5th harmonic current sensibility & & .0010 & & $5.6 \times 10^{-4}$ & & $2.2 \times 10^{-4}$ \\
\hline 5th harmonic EMF sensibility. & & $4.3 \times 10^{-6}$ & & $19 \times 10^{-6}$ & & $2.8 \times 10^{-6}$ \\
\hline Power sensibility. & & .0021 & & .045 & & .032 \\
\hline Resonance effect......... & & 39 & & 116 & & 2700 \\
\hline Ratio of back to impressed EMF. & & $78 \%$ & & $97.5 \%$ & & $87 \%$ \\
\hline Resonance range (current). & & $1.1 \%$ & $.14 \%$ & $.10 \%$ & $.10 \%$ & $.12 \%$ \\
\hline Resonance range (EMF)... & & $2.5 \%$ & & $9 \%$ & & $4.6 \%$ \\
\hline Time constant (current). & & .2 & & 1.7 & & 2.5 \\
\hline Time constant (EMF)... & & .09 & & .045 & & .23 \\
\hline Moment of inertia... & & .012 & .014 & .015 & .024 & .020 \\
\hline Moment of damping... & & .12 & & .018 & & .016 \\
\hline Moment of restoration.. & & 4500 & & 5700 & & 7700 \\
\hline Moment of displacement. . & & $20 \times 10^{4}$ & & $14 \times 10^{4}$ & & $11 \times 10^{4}$ \\
\hline
\end{tabular}

Those values obtained from measurements of the current sensibility, the direct current sensibility, the electromotive force sensibility, the resonating frequency, and the resistance are placed in the second column, while those values obtained by direct measurement or by an independent method are placed in the first column. Where there are values in both columns, the first serves as a check on the theory. Additional data for galvanometer No. 2 are given by Figs. 3 and 5 , and for galvanometer No. 3 by Fig. 4.

II The units used in this table are the same as given on page 359 , that is, the unit of amplitude of the vibration is a millimeter broadening of the image of a source of light I meter in front of the mirror; the unit of current is the micro-ampere and the unit of electromotive force is the micro-volt. 
The generator used in obtaining most of the data given was directly connected to a motor operáting in synchronism ${ }^{12}$ with a tuning fork whose frequency could be varied over a wide range by weights and continuously over a range of about $\mathrm{I} .5$ per cent by a spring. This gave the necessary control and regulation for the work at and near roo cycles. The electromotive force and current of three times this frequency was obtained from another generator whose speed was neither very constant nor definitely known.

\section{CHARACTERISTICS OF THE INSTRUMENTS.}

An inspection of the curves or a comparison of the constants will show that resonance has a marked effect upon the amplitude

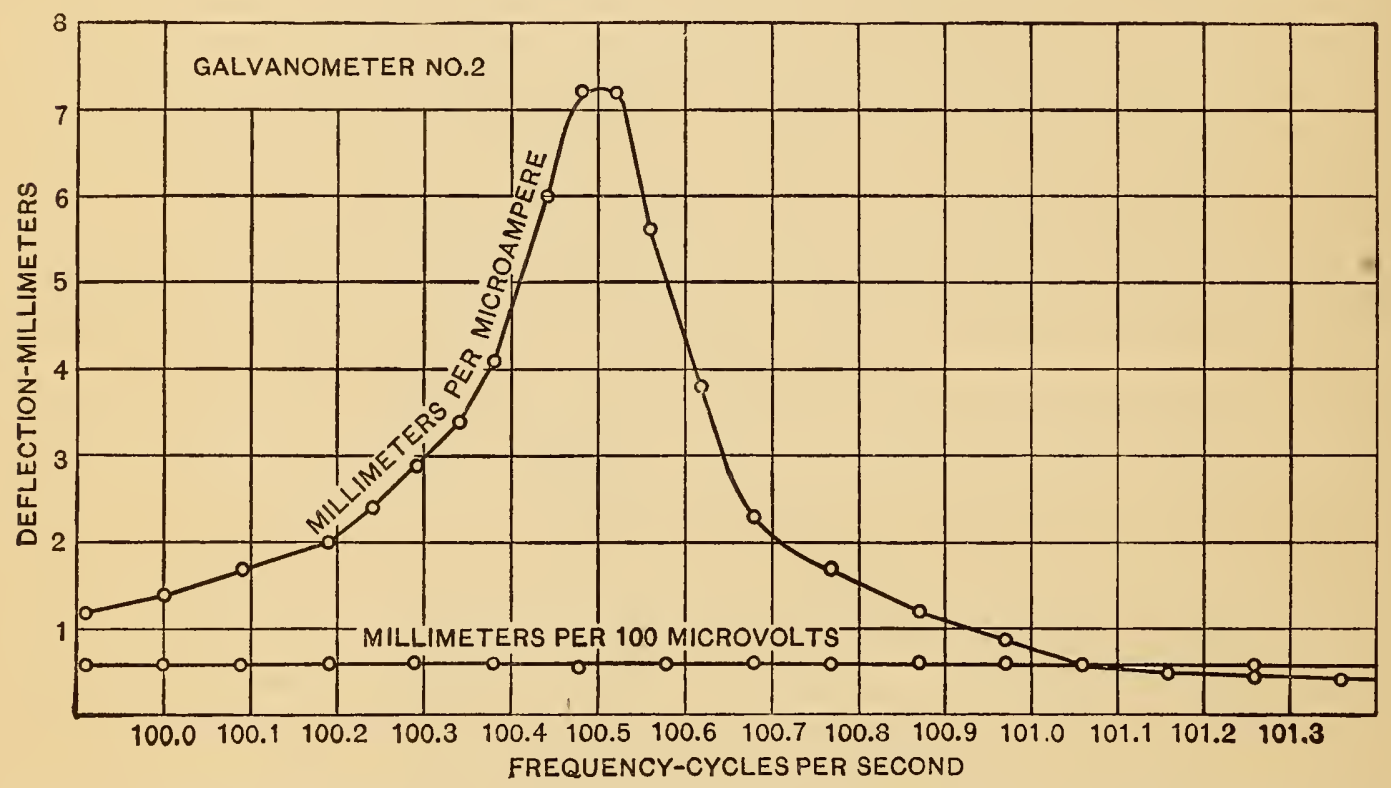

Fig. 2.-Showing the Effect of Tuning on Amplitude of Vibration with Constant Current and Constant Electromotive Force.

of the vibration. It seems to have been the idea that the effect of resonance ${ }^{13}$ was to increase the sensibility by about 100 times.

Taking the ratio of the alternating to the direct current sensibility gives: for No. I, 83; for No. 2, 730; and for No. 3, 7600. If, however, by the effect of resonance we mean the ratio of the amplitude produced by a certain amount of power supplied at the resonating frequency to the deflection produced by the same amount

${ }^{12}$ Hough and Wenner, Physical Review, 24, p. 535; 1907.

${ }^{13}$ M. Wien: Ann. der Physik, 309, p. 443; 1901.

Campbell Phil. Mag., 14, p. 497; 1907. 
of power supplied by direct current, these figures are too large, since no account has been taken of the back electromotive force.

A matter closely associated with the resonance effect is the change in the ratio of the amplitude of the vibration to the current, with changes in the frequency of the latter. This ratio in the neighborhood of the resonating frequency is shown by Fig. 2, for galvanometer No. 2 , and it will be seen that the ratio has a sharp maximum at 100.5 cycles per second and falls to one-half for a change of 0.14 per cent in the frequency.

It will also be noticed that a change of 0.5 per cent from the resonating frequency results in no noticeable reduction in the ratio of the deflection to the impressed electromotive force. Fig. 3 shows the ratio of the deflection to the electromotive force at

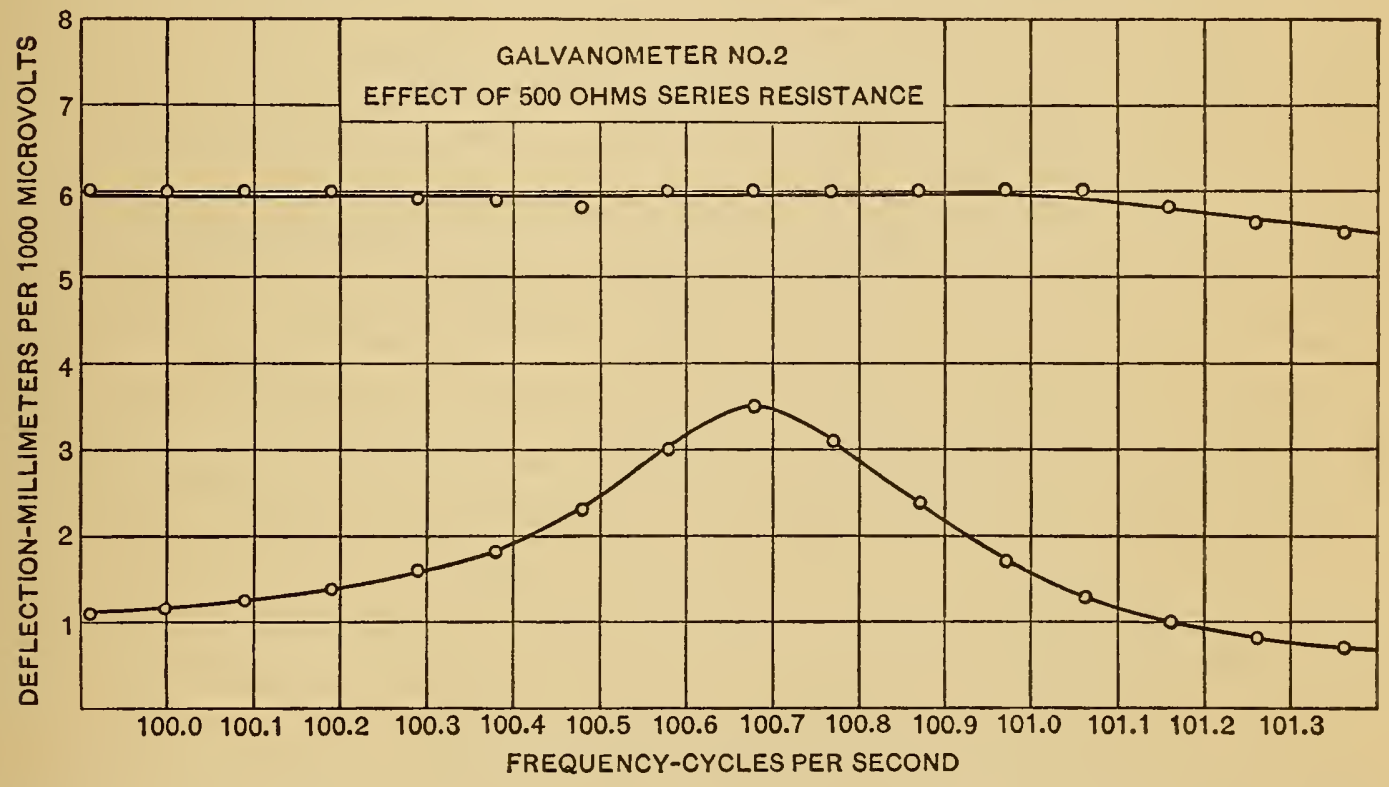

Fig. 3.-Showing Effect of Resistance in Decreasing Amplitude of Vibration with Constant Electromotive Force in the Circuit.

the terminals of the instrument and when 500 ohms are placed in series. The 500 ohms in series makes the resistance of the circuit I 8 times larger, yet at the resonating frequency the ratio of the deflection to the electromotive force is reduced by only about 45 per cent. The reduction calculated from the constants given in the table is about 32 per cent. It is evident from the behavior of this instrument that when it is used in bridge work with a constant impressed electromotive force the resistance of the galvanometer circuit, if under 300 ohms, has but little effect upon the sensibility. 
The effect of air damping on galvanometer No. 3 , is shown by Fig. 4. It will be noticed that the air reduces the resonating frequency by about 0.75 per cent. This change in frequency is accounted for if we assume that the system drags with it a quantity of air whose moment of inertia is equal to that of a sphere of air having a radius slightly larger than the radius of the disk. It is also to be noticed that the removal of the air makes the resonance curve much sharper. The effect which is of real importance is the reduction of the sensibility by the air friction.

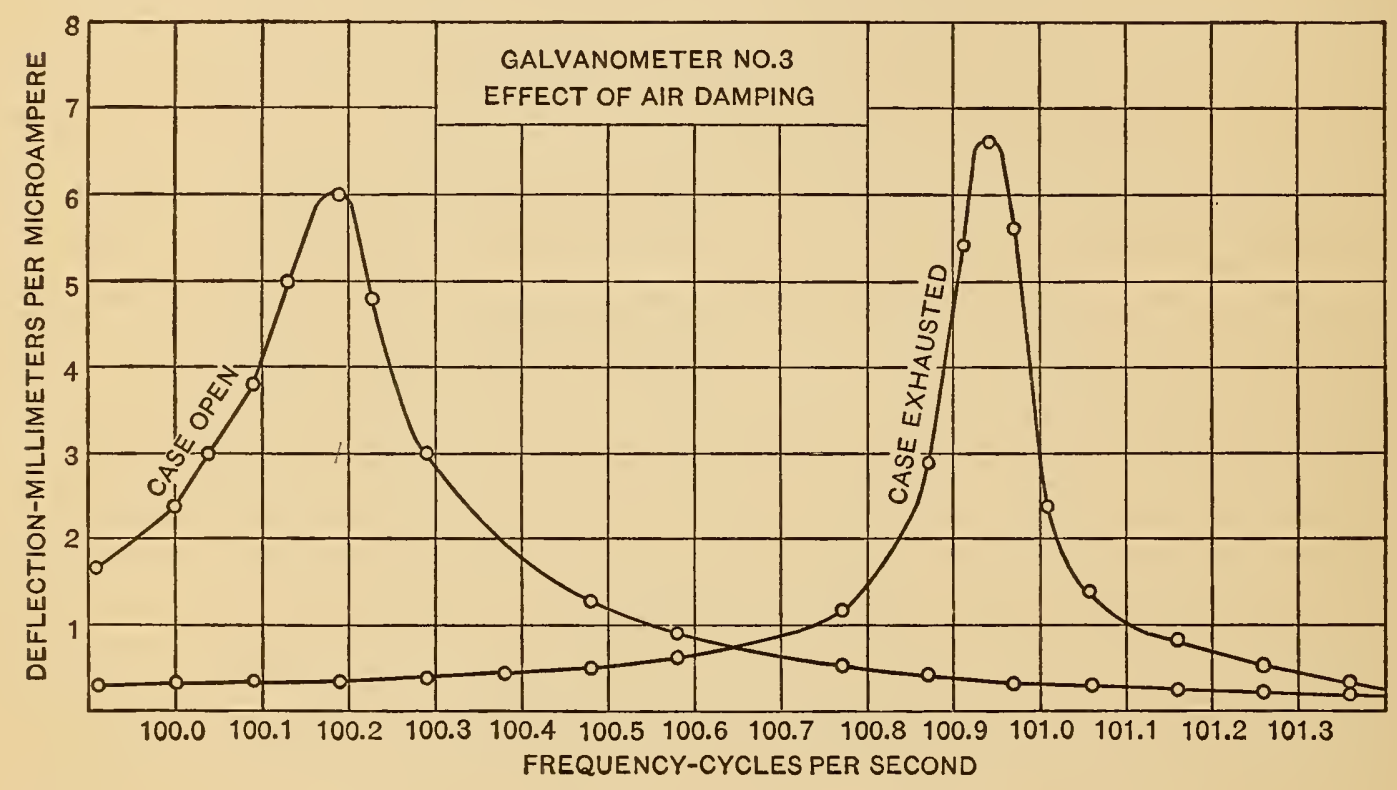

Fig. 4.- Showing Changes due to A ir Damping, in Resonating Frequency, Sharpness of Tuning, and Current Sensibility.

Since the back electromotive force is proportional to the amplitude the power supplied per microampere is, according to the curves, about 9 per cent less when air damped. For the same deflection, then, the power supplied, when air damped, is about I I per cent greater. While the adjustment of the instrument was changed between the time when the constants were determined and when the data for the curves were taken yet we may use, without appreciable error, the power sensibility given. This shows that the power in micro-microwatts necessary to maintain the vibration is 3 I times the square of the amplitude. Taking i I per cent of this gives as the power dissipated in air damping 3.5 times the square of the amplitude. 
Fig. 5 shows for galvanometer No. 2 the effect of a large inductance on the ratio of the amplitude of the vibration to the electromotive force. The inductance used was the 480 -volt winding of a 600 -watt transformer and the increase in the ratio was 125 per cent. While the resonating frequency was 100.52 , as shown in Fig. 2, yet the maximum amplitude of vibration occurred with a frequency of ror.o6 cycles per second. The effect observed was much more marked with an inductance about a quarter as large. No effort was made to get an inductance of the most suitable value.

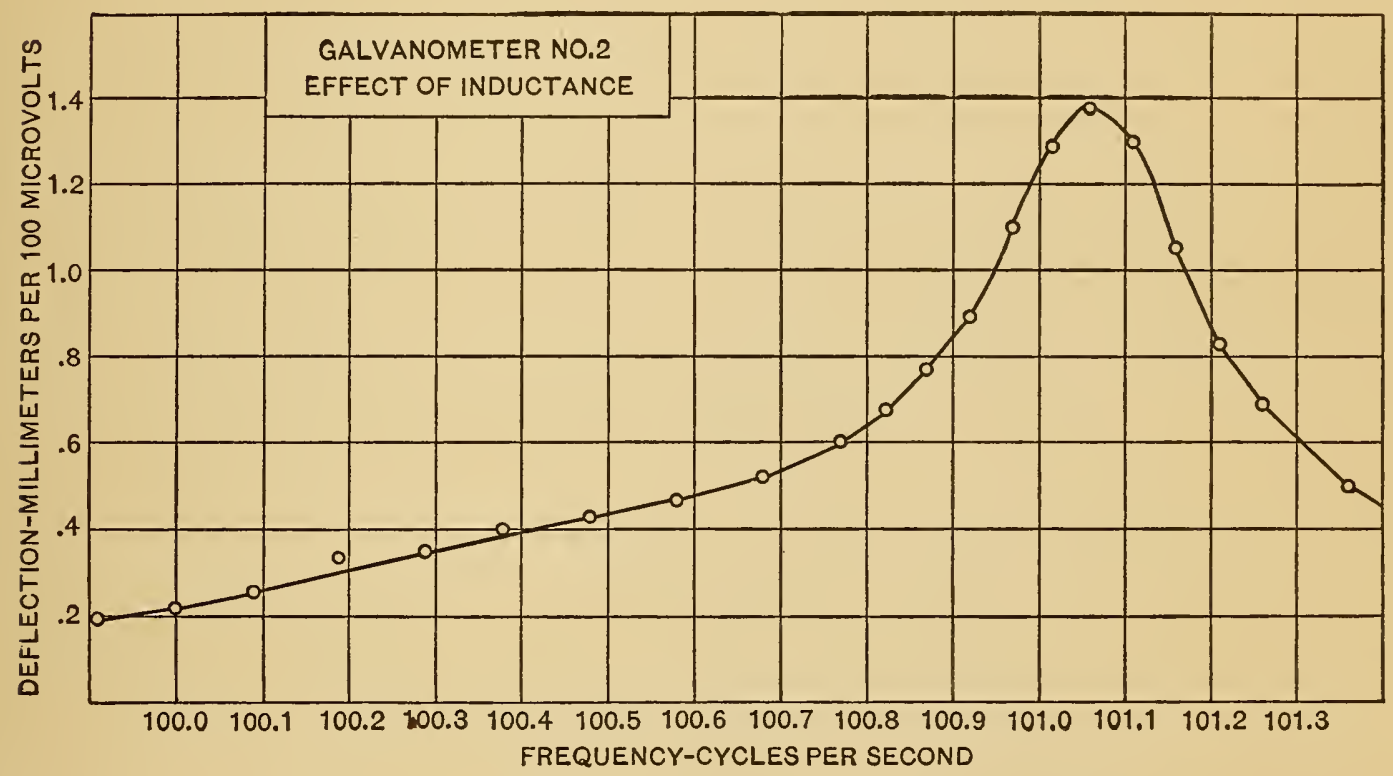

Fig. 5.-Showing Shift of Apparent Resonating Frequency and Increase of Amplitude of Vibration due to Inductive Circuit. Resonating Frequency 100.52, Apparent Resonating Frequency 101.06. Amplitude with Practically Noninductive Circuit .6 $\mathrm{mm}$ per 100 Microvolts.

\section{AN ILLUSTRATIVE PROBLEM.}

The consideration of the precision which may be attained in the balance of any one of the alternating current bridges will show the bearing of some of the above work on problems of this kind. On account of the simplicity of the equations, the problem chosen is the comparison of the product of a capacity and self-inductance with a frequency by the "series" method. Of course here only those factors which have to do with the precision of the balance will be considered. We are therefore concerned only with the case where the bridge is very nearly balanced and the galvanometer tuned to the frequency of the test current to be used. Most 
of the equations following are only approximate and can not be used except under these conditions.

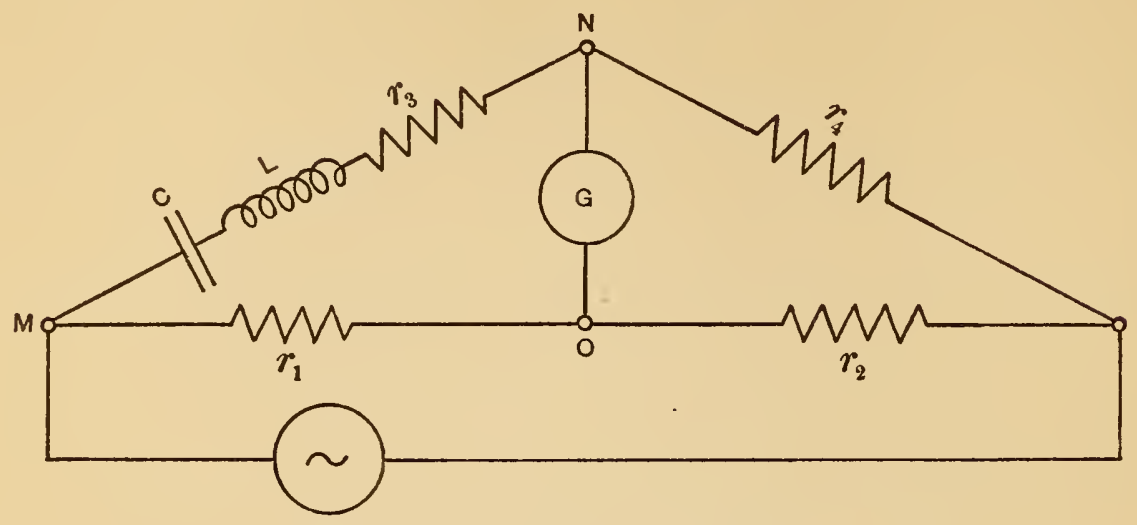

Fig. 6.-Series-Inductance Bridge.

The arrangement of the circuits is shown in Fig. 6, and the conditions for a balance are

$$
\frac{r_{1}}{r_{2}}=\frac{r_{3}}{r_{4}} \text { and } L p=\frac{\mathrm{I}}{C p} .
$$

For simplicity we shall take

$$
r_{3}=r^{\prime}(\mathrm{I}+a) \text { and } r^{\prime}=r_{1}=r_{2}=r_{4},
$$

also

$$
L^{\prime}=L(\mathrm{r}+b),
$$

where $a$ is the lack, in proportional parts, in the resistance balance, and $b$ is the lack, in proportional parts, of the inductance balance. Then if $E^{1}$ is the electromotive force impressed on the bridge the electromotive force between $M$ and $N$ and between $M$ and $O$ when the bridge is balanced is $1 / 2 E^{1}$. When the bridge is unbalanced by a resistance $r_{3}$ in place of $r^{\prime}$ and an inductance $L^{\prime}$ in place of $L$ the electromotive force between $M$ and $N$ is, if the galvanometer circuit is open,

$$
\frac{E^{1}\left[r_{3}+\sqrt{-\mathrm{I}}\left(L^{\prime} p-\frac{\mathrm{I}}{C p}\right)\right]}{r_{3}+r^{\prime}+\sqrt{-\mathrm{I}}\left(L^{\prime} p-\frac{\mathrm{I}}{C p}\right)}
$$

and since $L^{\prime} p-\frac{\mathrm{I}}{C p}=L b p$ this reduces to 


$$
\frac{E^{1}\left(r^{\prime}+r^{\prime} a+\sqrt{-I} L b p\right)}{2 r^{\prime}+r^{\prime} a+\sqrt{-I} L b p} .
$$

Substracting $1 / 2 E^{1}$ gives as the electromotive force of the galvanometer circuit

$$
E=\frac{E^{1}\left(r^{\prime} a+\sqrt{-\mathrm{I}} L b p\right)}{4 r^{\prime}+2 r^{\prime} a+2 \sqrt{-I} L b p},
$$

or since for an approximate balance $4 r^{\prime}$ is the only significant term in the denominator,

$$
E=\frac{E^{1}}{4 r^{\prime}}\left(r^{\prime} a+\sqrt{-1} L b p\right)
$$

If the points $N$ and $O$ are connected by a winding of any electrical instrument in which the ratio of the impressed electromotive force to the current is $m$ or simply by a conductor whose resistance is $m$ and if the circuit is practically noninductive, the current through the instrument is given by the equation

$$
I=\frac{E}{r^{\prime}+m}
$$

and the power delivered to the instrument is given by the equation

$$
w=\frac{E^{2} m}{\left(r^{\prime}+m\right)^{2}} .
$$

$w$ is a maximum when $m=r^{\prime}$ or

$$
w_{m}=\frac{E^{2}}{4 r^{\prime}} .
$$

If the points $N$ and $O$ are connected by a vibration galvanometer having a resistance approximately equal to its reactance, the current is given by the equation

$$
I=\frac{E-E_{b}}{r^{\prime}+\rho}
$$

where

$$
\begin{gathered}
E_{b}=D\left(E-r^{\prime} I\right) \\
\cdot E_{\dot{u}}=D E_{2} .
\end{gathered}
$$

and

$E_{2}$ is the electromotive force impressed at the terminals of the 
galvanometer. These three equations give

and

$$
I=\frac{E(\mathrm{I}-D)}{r^{\prime}(\mathrm{I}-D)+\rho}
$$

$$
E_{2}=\frac{E \rho}{r^{\prime}(\mathrm{I}-\bar{D})+\rho} .
$$

The power delivered to the terminals of the galvanometer is $I E_{2}$, therefore

$$
\text { - } w=\frac{E^{2} \rho(\mathrm{I}-D)}{\left[r^{\prime}(\mathrm{I}-D)+\rho\right]^{2}} .
$$

To get the power converted $w^{\prime}$, we may multiply $w$ by $D$ or

$$
w^{\prime}=\frac{E^{2} \rho\left(D-D^{2}\right)}{\left[r^{\prime}(\mathrm{I}-D)+\rho\right]^{2}} .
$$

The condition for the maximum value of $w^{\prime}$ is that

or

$$
D=\frac{\rho+r^{\prime}}{2 \rho+r^{\prime}},
$$

$$
\rho=r^{\prime}(\mathrm{I}-D) \text {. }
$$

Of these equations (29) gives the electromotive force of the galvanometer circuit, and this value substituted in (36) gives the current through the galvanometer or in (38) gives the power delivered to the terminals of the galvanometer. Equation (39) gives the power converted by the galvanometer and equations (40) and (4I) the condition under which this power is a maximum. Where a transformer is to be used, equation (4I) serves to determine the ratio which makes the sensibility of the whole combination a maximum. Since in well-designed and constructed instruments the power necessary to maintain a vibration of any specified amplitude depends only slightly upon the resistance or the ratio of the back to the impressed electromotive force (provided this ratio is over 75 per cent), the condition for maximum power converted is at least very nearly the condition for a maximum sensibility of the combination. Equations (40) and (4I) will therefore be of service in determining conditions which will give the best results with a particular instrument. 
Let us now consider the case where the resistances of the bridge arms are each roo ohms, the self-inductance .25 henry, the frequency roo cycles per second, and the electromotive force impressed on the bridge 20 volts.

In order that the bridge may be balanced the capacity required is a little over ro microfarads. If the bridge is out of balance by I part in 100,000 , both in the resistance and in the self-inductance, the electromotive force of the galvanometer circuit is, according to equation (29), 93 microvolts and the maximum power which the galvanometer may receive is, according to equation (32), 22 micromicrowatts. If galvanometer No. 2 is used, it will be seen from its power sensibility that unit amplitude of vibration is the maximum which can be obtained.

According to equation (38), the power actually received by the galvanometer is 6.1 micro-microwatts, and this gives .52 as the amplitude of the vibration. From equation (36) we get as the current through the galvanometer .07 I microampere, and this multiplied by the current sensibility gives .52 as the amplitude of the

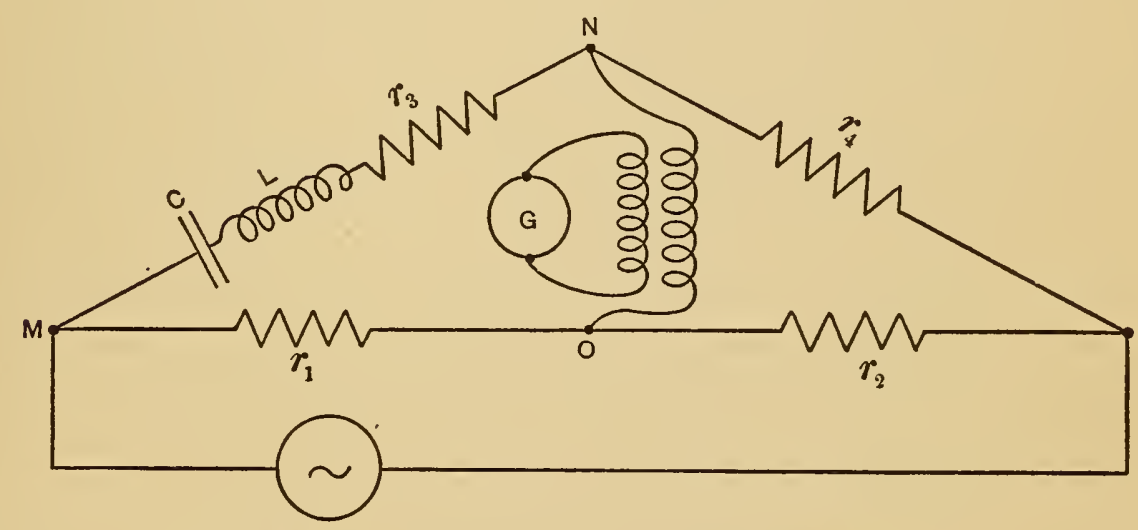

Fig. 7.-Series-Inductance Bridge showing transformer in Connection with the Galvanometer.

vibration. Again, using the electromotive force sensibility given by equation (23a), when $\rho$ is taken as the total resistance of the galvanometer circuit ( 30 ohms), and multiplying by the electromotive force, 93, we get the same deflection.

If, however, we use a transformer connected as shown in Fig. 7 , we see from equation (22a) that the step-up ratio of the transformer should be a little less than 3.5. Using 3.5 as the value of $X$ in equation (26) gives .0105 as the electromotive force sensibility 
of the combination and this multiplied by the electromotive force 93 gives .98 as the amplitude of the vibration. This, we have just seen, is about the maximum value attainable with this galvanometer.

The use of a transformer in this way, in effect, reduces the.resistance of the galvanometer inversely as the square of the step-up ratio without changing its power sensibility or the ratio of the back to the impressed electromotive force. Reference to equation (4I) shows that in effect the resistance of the combination of transformer and galvanometer should be 2.5 ohms to receive the maximum power available. Since the resistance of the galvanometer is 30 ohms (that of the transformer is assumed to be negligible), the step-up ratio should be $\sqrt{12}$. Taking this as 3.5 or 2.45 as the effective value of $\rho$, gives from equation (38) 22 micro-microwatts as the power received by the galvanometer, and from the power sensibility we get again .98 as the amplitude of the vibration.

For a frequency of 300 the impedance of the arm containing the self-inductance and capacity is large in comparison with that of the other arms. In calculating the current through the galvanometer, we may consider this arm to have an infinite impedance, and, further, since the back electromotive force is so small in comparison with the impressed electromotive force, it need not be considered. The current, then, through the galvanometer is approximately the same as would flow through a resistance of $\mathrm{r} 30$ ohms in parallel with roo ohms and with the combination in series with $100 \mathrm{ohms}$ when the electromotive force is equal to that impressed on the bridge. This gives

$$
I_{3}=\frac{E_{3}}{350} .
$$

If, then, the electromotive force impressed on the bridge has a $3 \mathrm{~d}$ harmonic amounting to only I per cent, $I_{3}$ will be 570 microamperes. Multiplying this by the current sensibility for the third harmonic we see that we have a third harmonic vibration of about the same amplitude as produced by a lack of balance of one part in one hundred thousand for the fundamental. Under these conditions, then, the use of a test voltage having a $3 \mathrm{~d}$ harmonic of I per cent limits the precision to about one part in one hundred thousand. 
If a transformer having a step up ratio of three and one-half is used, the $3 \mathrm{~d}$ harmonic current is reduced to about one-third of the former value, while as we have seen the sensibility of the combination to the fundamental is about doubled. This extends the precision of the balance to two parts in one million. It is thus seen that in certain cases the use of a transformer not only increases the sensibility of the combination but also permits the use of a test electromotive force having larger harmonics. If the relations given above for the use of a transformer âre to hold with a fair approximation, it is necessary that the secondary have a reactance large in comparison with $\mathrm{I} \div(\mathrm{x}-D)$ times the resistance of the gaivanometer, and that the resistance of the windings be small in comparison with the resistance of the circuits. In calculating the reactance it should be remembered that the permeability of the iron core is probably not over 200 for the very low magnetizing force used. For a frequency of IOO, a 2-kilowatt 6o-cycle "potential" transformer having the secondary wound for 15 times as many volts as the galvanometer has ohms will generally fulfill the conditions sufficiently well to give results within 20 per cent of those calculated from the equations. If bridges of different resistances are to be used the primary winding should be in sections to give the different ratios needed.

\section{DESIGN.}

While it is not our purpose to discuss fully, in this paper, the problem of the design of vibration galvanometers, yet a brief consideration, from this standpoint, of the theory and data given above will bring out a few of the principles involved. The consideration will be limited to instruments to be used in circuits of fairly low resistance, generally under 500 ohms and low electromotive force. This is the condition met with in alternating current bridge work which at the present constitutes the main field in which the vibration galvanometer is used. The instrument may be connected directly in series with the circuit, or to the secondary winding of a transformer the primary of which is connected in series with the circuit.

When the galvanometer is connected directly in series with the circuit, the relation between the amplitude of the vibration, the 
impressed electromotive force, and the various intrinsic constants of the instrument is given by equation (9). It should be noticed that the an plitude is a maximum for a particular value of the moment of displacement, that is when

$$
\psi^{2}=r \beta
$$

This is really the same condition as is given by equation (40) and both signify that the mechanical power received by the moving system is a maximum when the back electromotive force is equal to one-half the electromotive force impressed on the circuit. With galvanometer No. 2 used on a bridge having a resistance of roo ohms in each arm it will be seen that $\psi^{2}$ is about eight times as large as $r \beta$. As $\psi$ is proportional to the field strength of the magnet, it follows that a reduction of 60 per cent in the field strength results in an increase in the sensibility of the combination of about 70 per cent. A comparison with equation (Io) shows that this change results in a decrease of about 40 per cent in the power sensibility. Where $\psi^{2}=r \beta$ equation (9) takes the form

$$
\phi=\frac{E}{p \sqrt{2 r \beta}} .
$$

As $r$ is made up of the resistance of the galvanometer and the arms of the bridge in a series parallel combination but little is to be gained by making the resistance of the galvanometer excessively small. In inductance and capacity bridges $p$ is generally fixed from other considerations, so we have $\beta$ as the only factor under this condition, which materially affects the sensibility of the combination. The power necessary to maintain a vibration of a given amplitude is proportional to the moment of damping. The design then should be such that the back electromotive is approximately equal to one-half of the electromotive force impressed on the total galvanometer circuit, and the mechanical power necessary to maintain at the chosen frequency a vibration of any particular amplitude should be as small as possible. It is necessary also to take into consideration the resolving power of the optical system to be used, as this often limits the amplitude of vibration which may be detected. Where the galvanometer is to be used regularly with a transformer the ratio of the back electromotive force to that impressed at the 
terminals should be large, though there is little to be gained by making it over 90 per cent. Here, as before, the moment of damping, or the mechanical power necessary to maintain at the chosen frequency, a vibration of any particular amplitude, when the circuit formed by the winding is open, should be as small as possible.

It will be seen that the moment of inertia of the moving system does not affect directly the sensibility. It does, however, affect very materially the resonance range and the sensibility to the various harmonics. Where the balance depends upon the frequency or the wave-form the moment of inertia should be relatively large. On the other hand where the balance is independent of the frequency and especially if no special device is available for controlling the frequency, the moment of inertia should be very small to make the resonance range large.

We have seen (page 386 ) that the power lost by air friction by galvanometer No. 3 for a vibration of unit amplitude is 3.5 micromicrowatts. If we assume that 75 per cent of this is lost by friction and pressure against the mirror we see that the power sensibility of a galvanometer having a frequency of 100 , a mirror $\mathrm{I} \mathrm{cm}$ in diameter and operating in air is limited to .4, or about to times the sensibility of galvanometer No. 2 .

The sensibility may be made higher than this by changing the shape and reducing the surface of the moving system, yet if a very marked improvement is to be made in the sensibility it may be necessary to do away with the air damping by placing the instrument or at least its moving system in a vacuum.

\section{SUMMARY.}

I. The importance of the electromotive force developed by the relative motion of the magnet and the winding of a galvanometer is pointed out and the general theory of the vibration galvanometer is developed.

2. The fundamental equation is stated and, under certain conditions, a solution derived giving the various working constants in terms of the intrinsic constants.

$13260-10-6$ 
3. The advantage of using a transformer with an instrument developing a relatively large back electromotive force is shown.

4. The double period sometimes observed is shown to be due to an unsymmetrical system.

5. A method of tuning is described which is more sensitive than the method generally used and which is applicable in some other cases where the vibration is forced. This method consists in adjusting the frequency of the driving force or the moving system so that the phase angle between the driving force and the displacement produced is $90^{\circ}$.

6. Equations are derived giving the intrinsic constants in terms of five easily measured quantities, viz, the resistance, the directcurrent sensibility, the current sensibility, the electromotive-force sensibility, and the resonating frequency.

7. Intrinsic and working constants for three instruments are given, some of which were determined by two independent methods and thus serve as checks on the theory.

8. The principles to be followed in the design of a high-sensibility instrument for bridge work are developed, viz: (a) On account of the back electromotive force, heretofore overlooked, the resistance of the galvanometer should be very much less than the resistance of the bridge; $(b)$ the back electromotive force should be one-half of the impressed electromotive force; $(c)$ the mechanical power necessary to maintain at the desired frequency a vibration of a unit amplitude should be as small as possible.

WASHINGTON, May 25, 1909. 